\title{
BRAIN DAMAGE AFTER FEBRILE CONVULSIONS
}

\author{
BY \\ MALCOLM FOWLER \\ From the Adelaide Children's Hospital, South Australia
}

(RECEIVED FOR PUBLICATION NOVEMBER 5, 1956)

Despite the suggestions of several writers on the importance of convulsive seizures in infancy as an aetiological factor in the development of cerebral palsies, little attention is given to this association in the standard texts in general medicine and pathology, or even in the more special treatises on diseases of the nervous system. It is not altogether surprising, therefore, that convulsions occurring in the course of febrile illnesses are often not treated with the gravity and urgency they deserve. Because convulsions are so frequent an accompaniment of relatively innocuous infections and in most cases leave the patient apparently normal, the paediatrician may regard both a convulsion of purely febrile origin and the resulting signs of nervous disease as common manifestations of some other intracranial process.

The following five cases are presented in the hope that the potentially lethal nature of convulsions will be more widely acknowledged.

\section{Materials and Methods}

The pathological material was obtained from necropsies performed at the Adelaide Children's Hospital during the years 1954 to 1956 inclusive. Before immersion of the intact brain pieces were removed for fixation in formolammonium bromide, and in two cases samples were kept for animal inoculation. After prolonged fixation complete coronal slices of the cerebral hemispheres and smaller pieces of brain-stem and cerebellum were embedded in celloidin and paraffin, and stained by Weil's method for myelin sheaths, with Nissl's stain, and by haematoxylin, counterstaining with both eosin and van Gieson's mixture. Frozen sections were used for the metallic impregnation of neurofibrils and astrocytes using Bielschowsky's and Cajal's methods, respectively.

\section{Case Reports}

Case 1. The patient, previously a healthy boy of 2 years, developed measles two days before admission. He had not been very sick but had been coughing a lot. On the day of admission he was found in his cot at 7.45 a.m. in a convulsive attack, blue and frothing at the mouth. This continued until 9.30 a.m. when the convulsion was controlled by ether anaesthesia in the
Casualty Department of the Adelaide Children's Hospital where his colour was described by the Admitting Officer as ' almost black'.

On examination in the ward his temperature was $103^{\circ} \mathrm{F}$., he was slightly cyanosed, with a cold skin on which there was a fading measles rash. The pupils were equal and reacted sluggishly to light. The fundi oculorum were normal. The fauces were moderately reddened, the right tympanic membrane was dull and slightly bulging. Air entry into the lungs was good but there were many rhonchi. The abdomen was normal. Reflexes were absent in the limbs. Slight neck stiffness was present for an hour after the convulsions had ceased.

SPECIAL InVESTIGATIONS. The cerebrospinal fluid was under a pressure of $100 \mathrm{~mm}$. of water, contained $28 \mathrm{mg}$. protein per $100 \mathrm{ml}$. and no cells. The white blood count was 24,000 per c.mm., of which $81 \%$ were polymorphonuclear leucocytes.

Course. In the evening of admission he went pale, with a blood pressure of $70 \mathrm{~mm}$. systolic and $40 \mathrm{~mm}$. diastolic. This collapse was treated by intravenous serum infusion. The next morning he had a further convulsive attack which lasted for 10 minutes. He was comatose from the time of his first convulsion, responding by groaning to painful stimuli, and died three days after admission.

Post-MORTEM Findings. The necropsy (No. 41/56) was performed 22 hours after death. The body was wellnourished and on the skin of the back and face were the remains of a macular rash. In the lungs were numerous haemorrhagic areas of consolidation and these on microscopic examination were confirmed as foci of confluent haemorrhagic bronchopneumonia. Staphylococcus aureus was cultured from the lungs. There was severe centrilobular necrosis in the liver, involving about one half the thickness of the lobule. The liver cords were disorganized, and the cells were intensely eosinophilic and shrunken, with pyknotic nuclei. Many cells were disrupted into granular fragments. There was no inflammatory reaction in the portal areas and there was no vascular congestion.

In the spleen there were large reactive centres and reduced numbers of lymphocytes in the Malpighian bodies.

Central Nervous System. No abnormality apart from much congestion was noted in the brain on naked-eye examination. Microscopically, however, there was a widespread necrosis of neurones. The affected cells 


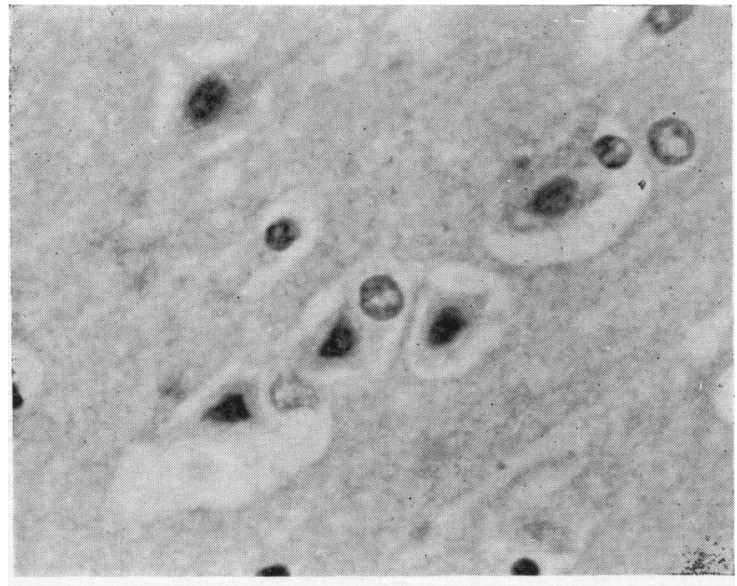

FIG. 1.

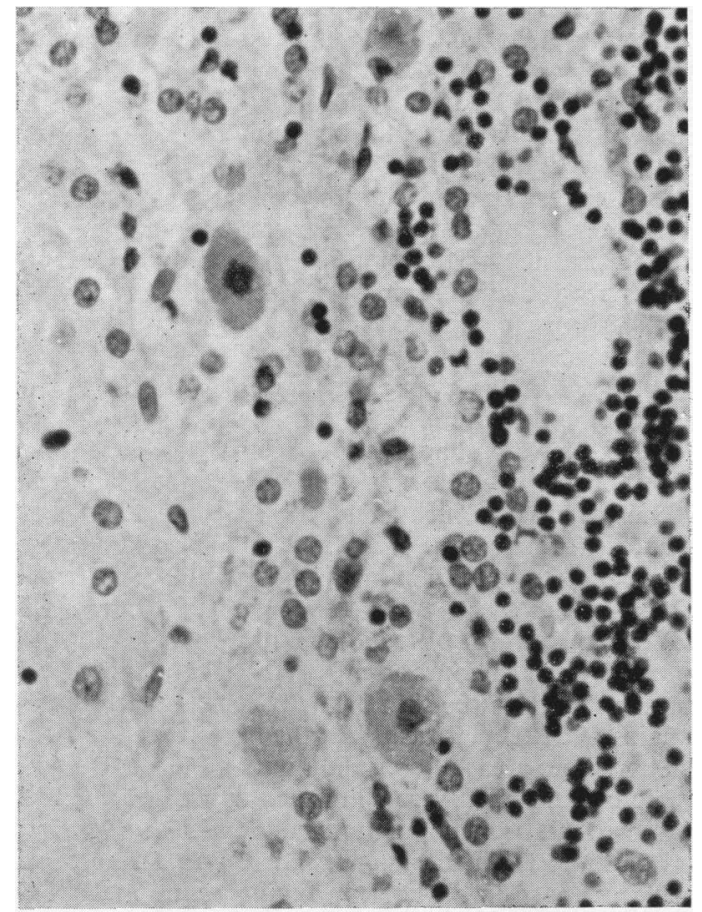

Fig. 2.

were shrunken, their cytoplasm was brightly eosinophilic, all Nissl material having disappeared, and the nucleus was small, irregular in shape and pyknotic (Fig. 1). Often the nucleolus was enlarged and eosinophilic. The blood capillaries in these areas were moderately or greatly dilated, but there was no haemorrhage or softening and only occasionally were a few small round cells seen in the perivascular space. In addition to neuronal damage, the astrocytes in the affected grey matter were also abnormal in that they failed to impregnate with gold although their nuclei did not look abnormal using

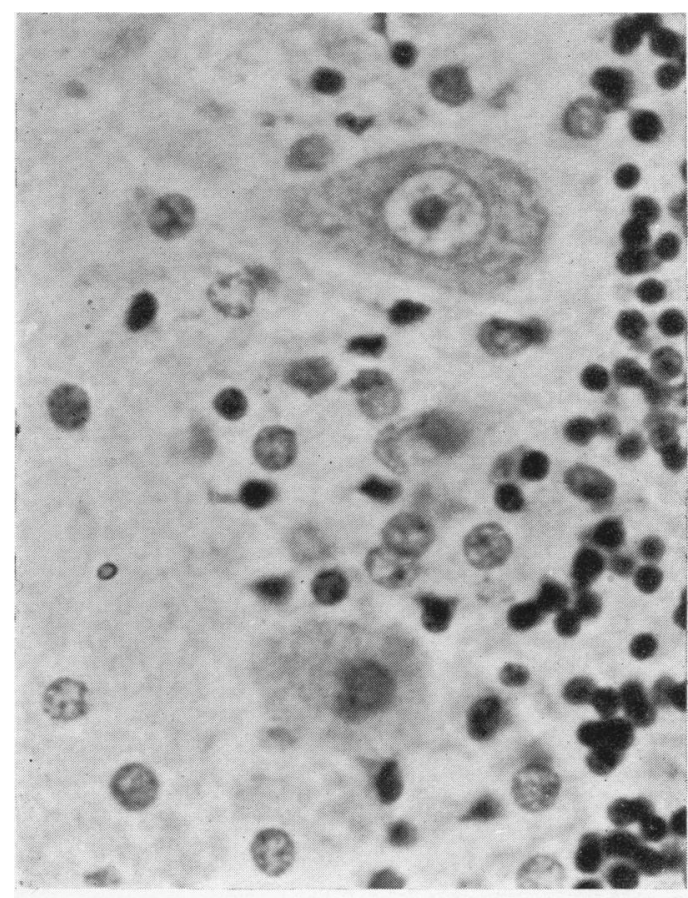

FIG. 3.

FIG. 1.-Case 1: frontal cortex showing shrunken neurones with pyknotic nuclei, and pericellular vacuolation. Haematoxylin and eosin $\times 700$.

Fig. 2.-Case 1: cerebellum showing shrunken Purkinje cells with pyknotic nuclei.

Haematoxylin and eosin $\times 450$

FIG. 3.-Case 1: cerebellum showing a normal and a necrotic Purkinje cell.

Haematoxylin and eosin $\times 825$.

haematoxylin stains. Astrocytes in the white matter underlying the cortex stained normally but those on the junction were incompletely impregnated and often appeared to have fragmented processes. In most areas there was much perivascular and pericellular vacuolation.

All parts of the cerebral cortex were involved but particularly the temporal and frontal gyri. The occipital cortex, the hippocampus and dentate fascia were damaged on both sides, but far more severely on the left side. The thalamus and amygdaloid nucleus on both sides showed an equally severe degree of neuronal 
necrosis, but the lesions in the caudate nucleus were more extensive on the left. The putamen and globus pallidus showed only pericellular oedema on the right but were severely damaged on the left. Small focal lesions were present in the left claustrum. In the cerebellum there was selective damage to the Purkinje cells, other cells of the cortex and the central nuclei being unaffected. On the whole there was not much damage in the cerebellum, but two areas involving several adjacent folia were present, one in the vermis, the other in one lateral lobe. In these foci the majority of the Purkinje cells were shrunken, with brightly eosinophilic cytoplasm and contracted densely staining nuclei (Fig. 2). Many had completely disappeared and others were merely faint outlines. Often a normal Purkinje cell would be found amongst degenerate neighbours (Fig. 3). In other parts of the cerebellar folia the Purkinje cells showed patchy chromatolysis.

Case 2. This child, a boy aged 1 year, was admitted with a two days' history of anorexia, drowsiness and running nose. The night before admission he had had a convulsion unrelieved by intramuscular phenobarbitone but stopped by ether anaesthesia. The attack lasted about half an hour. He had begun convulsing again in the morning at 6.15 o'clock and was still convulsing when first seen in the Casualty Department at 7 a.m. The child was very cyanosed. After treatment with phenobarbitone and ether the convulsions stopped at about 7.15 a.m.

On examination in the ward he was unconscious and pale, with cyanosed lips. His temperature was $104^{\circ} \mathrm{F}$. There was some residual intermittent twitching of the right arm and leg. The fundi oculorum were normal and the ear drums slightly reddened. There was no neck stiffness, and the thorax and abdomen were normal. The knee and ankle jerks were absent and the plantar reflexes were flexor.

SPECIAL INVESTIGATIONS. A sample of cerebrospinal fluid, taken immediately after admission, was not under increased pressure, and contained 14 white blood cells, and 1,060 red blood cells per c.mm., $60 \mathrm{mg}$. protein, and $35 \mathrm{mg}$. sugar per $100 \mathrm{ml}$. Further lumbar punctures on the fifth and eleventh days of his illness yielded fluid again contaminated with blood. The blood contained 21,000 white cells per c.mm. of which $80 \%$ were polymorphonuclear leucocytes. Radiological examination of the chest showed no sign of disease. Blood culture was negative and no abnormal organisms were found in the faeces. The urine was normal.

Course. Two days after admission the features of a right hemiplegia, previously indefinite, emerged clearly, the facial and peripheral musculature on this side being paralysed, with exaggerated tendon reflexes and extensor plantar response. His temperature was consistently at or above $103^{\circ} \mathrm{F}$. A few days before death the coma lightened enough for him to be able to respond by groaning to painful stimuli. He died on the twelfth day of illness in a state of dehydration, deeper coma and subnormal temperature.

Post-MORTEM Findings. The necropsy (No. 94/55) was performed 18 hours after death. There was a purulent right otitis media, and confluent bronchopneumonia. Yellow streaks were found in the pectoralis minor muscles. There was necrosis of all papillae in the left kidney, but of only one papilla in the right. All these lesions were confirmed microscopically, the muscle changes being those of necrosis and calcification, probably secondary to rupture during the convulsion. The pulmonary and renal changes were both very recent.

Central Nervous System. The parietal lobes, particularly on the left, were hyperaemic. On section the cortical grey matter of the left cerebral hemisphere was peppered with fine red dots. The dural sinuses were normal.

Microscopically the basic lesion was degeneration of neurones. In the most severely affected areas the nerve cells had disappeared but in other places many were still present and showed degenerative changes-shrinkage, loss of Nissl substance, acidophilia of the cytoplasm, pyknosis and enlargement of the nucleolus which was weakly acidophilic (Fig. 4). Coincident with loss of the nerve cell body was a great scarcity of axonal processes, and many showed beading and fragmentation. In the central white matter of the cerebral hemispheres myelin sheaths were finely beaded. In the affected grey matter microglial proliferation was obvious, sometimes as amoeboid forms, but more conspicuous was the pleomorphic rod-cell stage. Even in areas of cortex where degenerate neurones were still visible normal astrocytes could not be demonstrated by Cajal's gold impregnation method, except in the subpial layer where they were increased. Astrocytes were present in large numbers in the immediately subcortical white matter, and here they were impregnated clearly (Fig. 5). Abnormal forms could be seen in the junction between cortical grey and white matters and in the deeper parts of layer $I$ in the cerebral cortex (Fig. 6). The capillary vessels in the affected grey matter were extremely numerous and their endothelial and adventitial nuclei were very prominent (Fig. 7). There was a very occasional slight infiltration of lymphocytes around larger vessels. The meninges were thickened and contained an excess of macrophages.

The grey matter of the left cerebral hemisphere was very severely affected. The lesions in the cerebral cortex were diffuse and of approximately equal severity in all the frontal and temporal gyri, the anterior and posterior central gyri, the gyrus cinguli, the insula, the uncus and the fusiform gyrus. The hippocampal gyrus had lost layer III of its cortex for a short segment. There was almost complete loss of neurones in the hippocampus, particularly where its cortex enters the concavity of the dentate fascia. The granule layer of the dentate fascia showed much less outfall, exhibiting scattered small foci and segments of partial or complete loss. In the thalamus, putamen, caudate nucleus and amygdaloid nucleus only a few recognizable cells remained. The globus pallidus showed lesions of lesser severity although in one section all cells had been lost from its inner division. A small focal lesion was seen in the anterior perforated substance, and there were moderate numbers of such foci in the claustrum.

On the right side lesions were very much less pronounced. The cortex was normal, apart from damage 


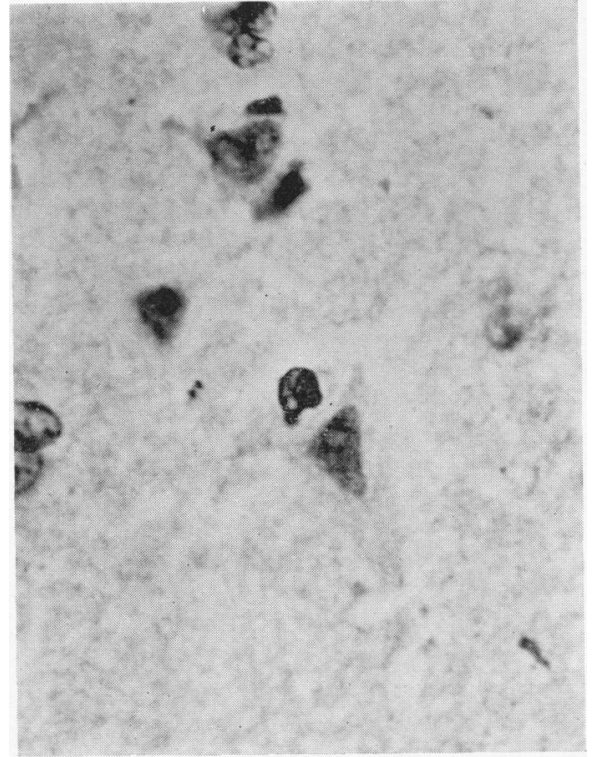

FIG. 4.-Case 2: frontal cortex showing shrunken neurone with pyknotic nucleus, enlarged pale nucleolus and faint cytoplasm.

Haematoxylin and eosin $\times 790$.

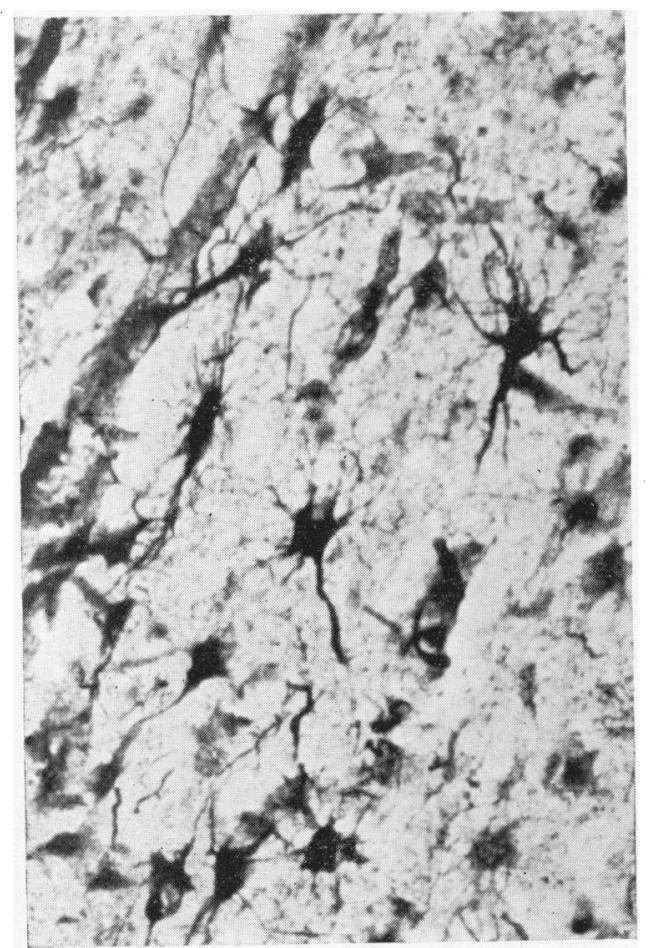

Fig. 5.-Case 2: numerous astrocytes in the white matter immediately beneath the frontal cortex.

Cajal's gold chloride $\times \mathbf{4 0 0}$.

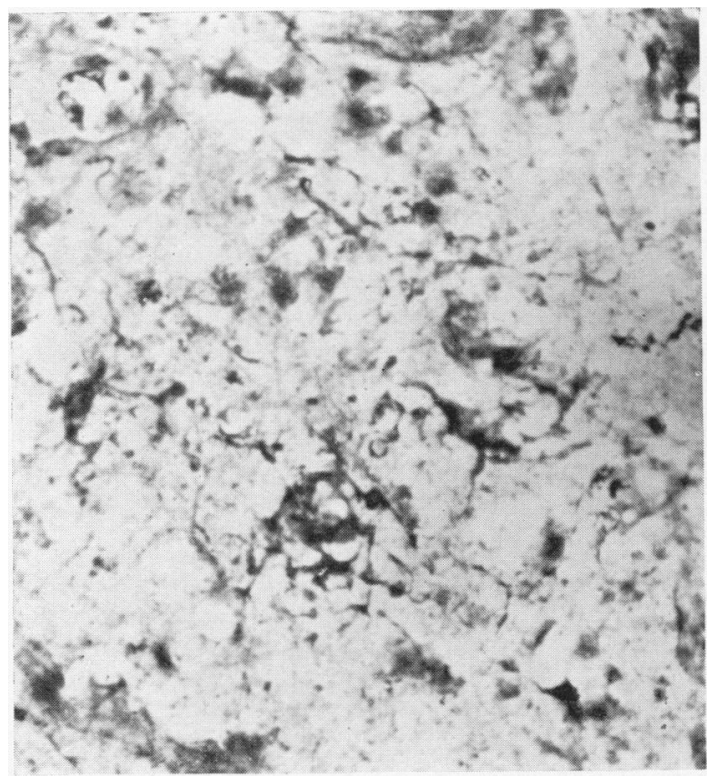

Fig. 6.-Case 2: abnormal astrocytes on the border of grey and white matters of the frontal cortex.

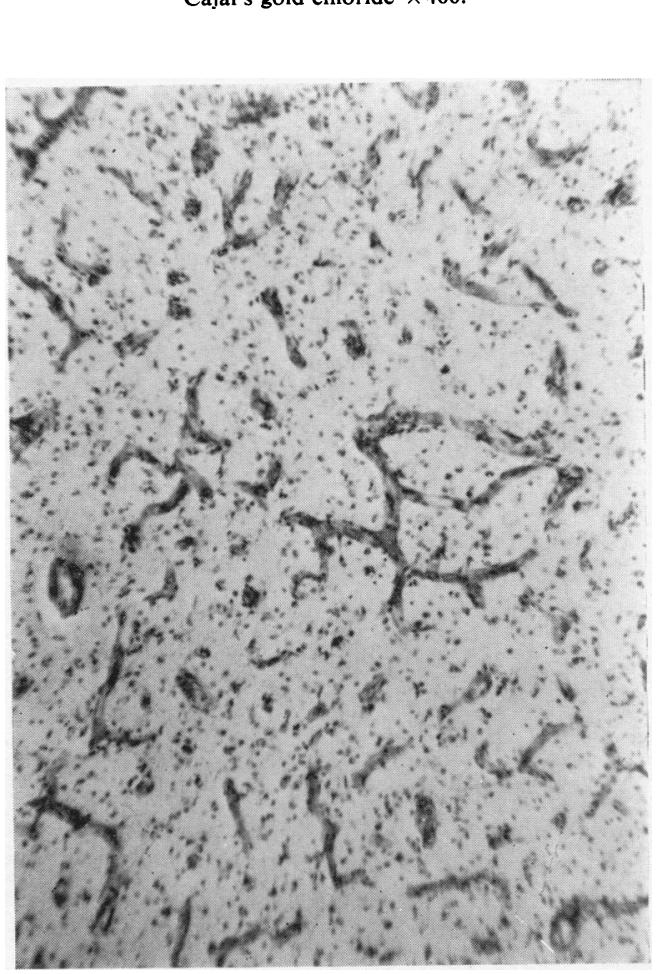

Fig. 7.-Case 2: temporal cortex showing capillary proliferation.

Haematoxylin and eosin $\times 90$ 


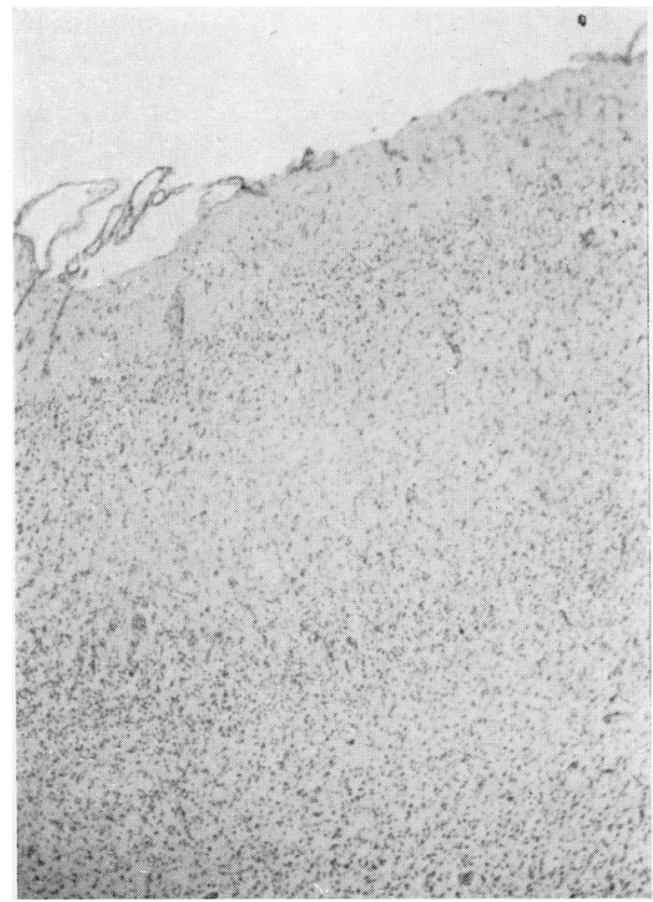

Fig. 8.-Case 2: insular cortex showing loss of neurones in layer III. Haematoxylin and eosin $\times 30$.

to the superior part of the superior temporal gyrus, inferior portion of the insular cortex and to the hippocampal gyrus, and in these portions layer III had been selectively damaged. There was moderate destruction of cells in the anterior nucleus of the thalamus, a small focal lesion in both the hippocampus and the central part of the dentate fascia. The putamen, globus pallidus, caudate nucleus, claustrum and the rest of the thalamus were normal.

Structures of the midbrain, medulla oblongata and cerebellum were normal.

In the cerebral cortex, layers III, V and VI were most severely damaged, and, as stated above, the neurones of layer III were sometimes involved alone (Fig. 8). Layer II often was visible when all deeper layers were completely disorganized.

Case 3. This girl, aged 21 months, had been irritable, feverish and anorexic for a week before admission. On the day before admission she had vomited once and retched several times. At 4.45 p.m. on the day of admission her mother had found her in a convulsive seizure involving all limbs; her skin was blue, cold and sweaty. The fit lasted an hour and was then followed by unconsciousness in which the child's respirations were slow and grunting.

On examination in the ward she was in unrousable coma and breathing irregularly. Her temperature was $102 \cdot 2^{\circ} \mathrm{F}$. and her pulse 160 . The eyes were deviated to the left and there was a fine nystagmus to the left. The pupils were moderately dilated, equal and reacted to light. The fundi oculorum were normal. The thorax and abdomen were normal. The legs were hypertonic, without tendon reflexes but with extensor plantar responses. The arms were flaccid, with active reflexes equal on both sides. There was a fine erythematous rash on the trunk and limbs. Four hours after admission she began to convulse and this fit lasted two hours.

SPECIAL INVESTIGATIONS. The cerebrospinal fluid was under a pressure of $260 \mathrm{~mm}$. of water, and contained 4 white cells per c.mm. and $22 \mathrm{mg}$. protein per $100 \mathrm{ml}$. The urine contained many granular casts. The white cells in the blood numbered 20,000 per c.mm., of which $62 \%$ were polymorphonuclear leucocytes. Neither lead, antimony, arsenic nor mercury was present in the gastric contents. The urine and blood showed no evidence of lead in abnormal amounts. Radiological examination of the skull and chest showed no abnormality. Blood culture was not done.

Course. She remained deeply comatose and her limbs became more spastic, the legs in extension, and she gradually developed opisthotonos. After two days at $102^{\circ} \mathrm{F}$. her temperature fell to below $100^{\circ} \mathrm{F}$. Two further examinations of the cerebrospinal fluid showed normal pressure and no increase in protein; 7 cells per c.mm. were present on one occasion, none on the other. The urine a week after admission was normal. No organisms of a pathogenic nature were isolated from the faeces. She died four weeks after admission in a state of decerebrate rigidity.

PosT-MORTEM FIndings. The necropsy (No. 13/56) was performed five hours after death. Apart from an early but widespread bronchopneumonia, confirmed microscopically, there were no significant changes except in the brain.

Central Nervous System. There was a slight increase in the space between the cerebral hemispheres and the skull. The frontal lobes were very pale and their convolutions slightly angular. The parietal and temporal lobes were very spongy to touch, and the grey matter of the temporal lobes (even after fixation) tended to fall away spontaneously under the most gentle handling, and was very granular in appearance, with the consistency of stiff porridge. The occipital lobes were firm and vascular by comparison. The central white matter concontained conspicuous capillaries but otherwise was normal. The cerebellum, brain-stem and dural sinuses were normal.

Microscopical examination revealed loss of neurones of variable degree throughout the cerebral cortex and basal nuclei.

In the most severely damaged areas of cortex all neurones had disappeared, resulting in a complete loss of stratification, and the grey matter tended to be fragmented, even in the celloidin sections (Fig. 9). In many areas the thickness of the cortex was already reduced and early tenting of the surface was recognizable. There was much astrocytic proliferation in the white matter immediately beneath the affected cortex, but the astrocytes in the grey matter, apart from those of the subpial 


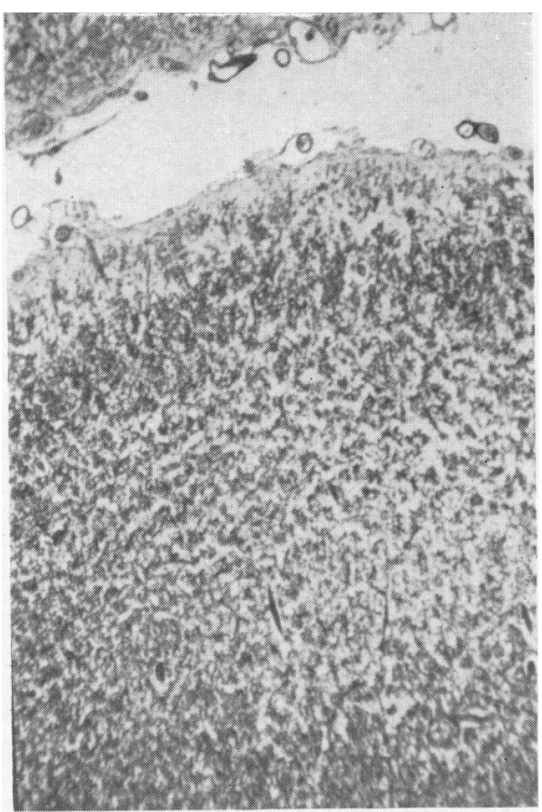

FIG. 9.-Case 3: temporal cortex showing fragmentation. Weil stain $\times 25$.

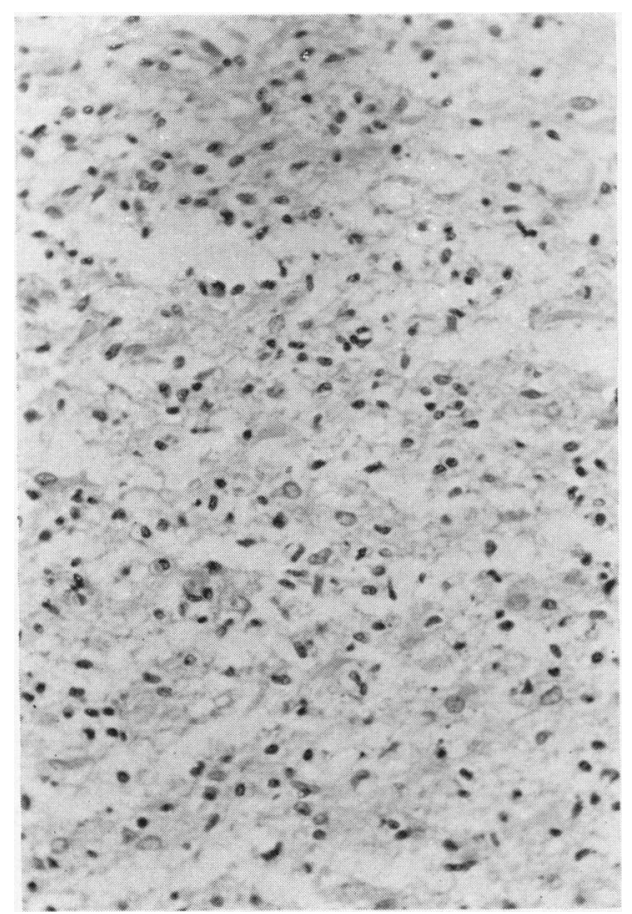

FIG. 10.-Case 3: caudate nucleus showing loss of neurones and microglial proliferation. Haematoxylin and eosin $\times 250$. layer, were fragmented and impregnated poorly. Axis cylinders were grossly reduced in number, a mere scattering of faintly impregnated fragments remaining. Many areas had suffered minimal loss, perhaps of only one cortical layer, and in this case astrocytic proliferation within the grey matter was marked. Microglia, mostly in the rod-cell stage, were often present in large numbers, except in areas of most severe damage (Fig. 10). Between these extremes all grades of neuronal loss were encountered. A few small strips of cortex were completely normal but the vastly greater part showed some lesions. Capillaries were slightly increased in damaged areas but there was no perivascular or meningeal infiltration by inflammatory cells.

Of the cortex, that in both temporal lobes suffered most severely, all neurones having been lost from all the gyri in the anterior portions of these lobes. Small segments of complete loss were scattered throughout other areas of cortex. All frontal gyri on both sides were severely affected, and lesions were also found in the anterior and posterior central gyri, the cingulate gyrus, insular cortex, the hippocampal gyri and the orbital gyri. A small area, about $1 \mathrm{~cm}$. long, of moderately severe cell loss was present in the cortex of the hippocampus and some lesser damage in the concavity of the dentate fascia. Layers II and IV were the most often preserved, and sometimes for long stretches all other layers had disappeared while these remained. Layers $\mathrm{V}$ and VI were on the whole the most severely and frequently involved.

The lateral and anterior parts of the thalamus were considerably damaged, particularly the anterior nucleus, and on one side the amygdaloid nucleus had lost nearly all its cells. The head of the caudate nucleus had suffered much loss on one side whereas only its mid-portion on the other contained lesions and these were very slight. The claustrum on one side showed a few focal areas of loss. The putamen, globus pallidus, substantia nigra, red nucleus and subthalamic nucleus on both sides were normal, nor were any changes found in the cerebellum or medulla. The choroid plexus, meninges and white matter were normal.

Case 4. Until the present illness at the age of 13 months, this patient had developed normally and was alert, intelligent and affectionate. One week before her illness she had not been herself, being restless and irritable after having a haemangioma on the temple treated with carbon dioxide snow. On the day before the convulsion she was hot and drowsy. The next day she had a temperature of $103^{\circ} \mathrm{F}$. in the morning but ate a good lunch and had a normal motion. Apart from being hot and having a foul breath she was normal at 3 o'clock in the afternoon. At 4 o'clock she gave a cry and went into a convulsion which lasted two and a half hours, during which her colour was bluish-grey. The doctor took her temperature just after 4 p.m. and found it to be $105^{\circ} \mathrm{F}$. She remained unconscious, with intermittent clonic movements all night but her colour was normal. The next morning she passed foul fluid motions full of blood and mucus. She was admitted unconscious to a private 
ward. The sample of cerebrospinal fluid taken just after admission was normal. Six weeks later when she was transferred to a public ward, spasticity in flexion of both arms and slight spasticity of the right leg were present, and she lay in bed showing no awareness of her surroundings.

Ten days after admission her temperature rose to $105^{\circ} \mathrm{F}$. and the left tympanic membrane bulged a little. This settled down with penicillin treatment but there was intermittent pyrexia over the next month, occasional crepitations being heard in the lungs. Six weeks after admission she had a typical attack of measles and three days after the rash had faded her temperature rose again, as a result of a right otitis media, both drums at this stage being opened under anaesthesia. From the pus obtained pneumococci were cultured. Her temperature fell temporarily, only to rise with the appearance of widespread crepitations in the lungs. Her general condition deteriorated and death occurred 18 weeks after the onset of her illness. She had remained unconscious throughout.

Special Investigations. Apart from examinations of the cerebrospinal fluid at the beginning of her illness two further samples, both normal, were taken at three weeks and 11 weeks after the onset.

The Mantoux reaction was negative. Blood examination showed little significant abnormality, except for a raised white blood count $(21,000$ per c.mm.) on one occasion. Sera collected four weeks and 10 weeks from the onset of the illness gave negative complement-

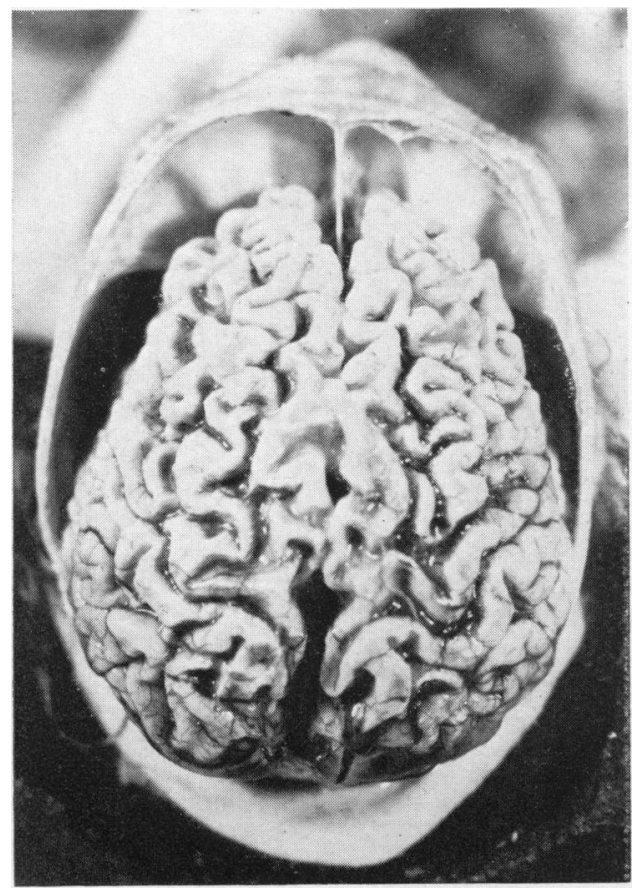

Fig. 11.-Case 4: atrophy of convolutions of frontal and anterior parietal lobes.

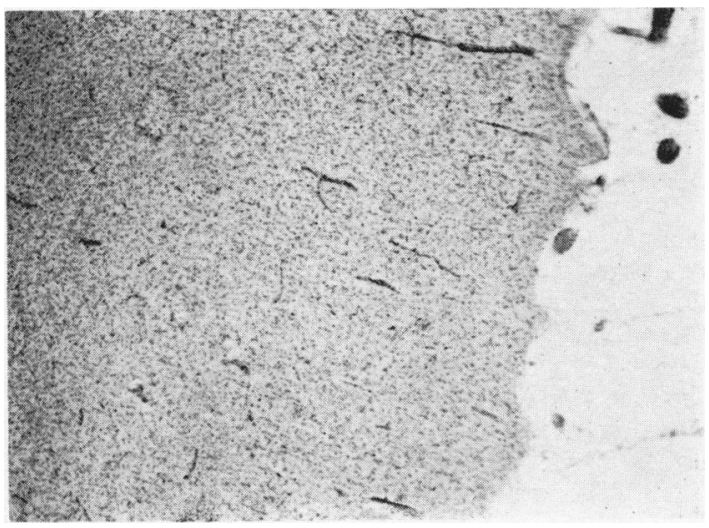

FIG. 12.-Case 4: frontal cortex showing loss of architecture and surface irregularity.

Haematoxylin and van Gieson $\times 25$.

fixation reactions against mumps, lymphocytic choriomeningitis and Murray Valley encephalitis viruses.

Post-MORTEM Findings. The necropsy (No. 32/54) was performed two hours after death. Apart from general wasting of the body there was a bilateral purulent otitis media and mastoiditis, and terminal bronchopneumonia.

Central Nervous System. Both frontal lobes and the post-central gyri were shrunken with a corresponding increase in cerebrospinal fluid over them. The gyri were narrow and angular and were separated by wide sulci (Fig. 11). The temporal and occipital lobes and cerebellum looked normal. Coronal slicing of the brain in the affected areas revealed irregularity and reduction in thickness of the cortical grey matter and mild reduction in bulk of the central white matter. The basal ganglia were normal.

Microscopic examination showed a mild cellular thickening and increase of collagen fibres in the arachnoid membrane, but no inflammatory cells. Neurones had disappeared in greater or less degree throughout the cortex of the gyrus cinguli, the frontal gyri, anterior central and posterior central gyri. The mid-portion of the caudate nucleus, the lentiform nucleus, the hippocampus, amygdaloid nucleus, mammillary bodies, lateral part of the thalamus, brain-stem and cerebellum, and the temporal gyri were normal. The head of the caudate nucleus, midbrain, occipital cortex and other parts of the parietal cortex and thalamus were not examined microscopically. Large parts of the cortex were extremely thin, with almost complete loss of neurones and with surface tenting (Fig. 12). In areas of lesser severity the layering of neurones was severely disturbed, layers III and V apparently suffering most. Even when other layers had completely disappeared layer II was often still recognizable. No Betz cells were seen. Coincident with the disappearance of neurones was an extreme reduction in nerve fibres, and an increase in astrocytes, particularly in layers V and VI and in the adjacent white matter. In the white matter there was a diffuse pallor together with vacuolation of remaining 
myelin sheaths and there were numerous grape-like bodies. Capillaries had increased slightly in number, but were not accompanied by any infiltration of inflammatory cells in their adventitial zones.

Case 5. This girl was first admitted at the age of 10 months for treatment of scurvy, having had no vitamin additions to her diet of cows' milk and 'farex'. After treatment with ascorbic acid and iron for a fortnight the child was discharged much improved. The following day, however, she was readmitted at 1 p.m. having been convulsing since 7 a.m. On examination she was convulsing and had a temperature of $105^{\circ} \mathrm{F}$. The convulsions involved the left side more than the right. The fundi oculorum and pupils were normal. There was no neck stiffness nor any abnormality noted in the chest, abdomen or limbs. Following treatment she came out of the convulsive state but was comatose, responding to painful stimuli by withdrawal, and the limbs were spastic.

SPECIAL INVESTigations. The haemoglobin value was $8 \mathrm{~g}$. $\%$ and the white blood cells numbered 28,000 per c.mm., $92 \%$ being polymorphonuclear leucocytes. The urine contained numerous granular casts. The cerebrospinal fluid, which was not under increased pressure, contained $21 \mathrm{mg}$. of protein per $100 \mathrm{ml}$., and no cells. Culture of the faeces yielded a Salmonella group C organism. Blood culture was negative. The Wassermann reaction was negative. Blood taken five weeks after the illness began had no complement-fixing activity against mumps and Murray Valley encephalitis viruses.

Course. Her temperature remained elevated until a week after admission when it returned to normal, and at this time a further culture of the faeces was negative for Salmonella organisms. The child's state of consciousness, however, improved very little. She lay in bed unaware of her surroundings, often breaking into spasms of high-pitched crying. She remained spastic in all limbs, the arms being held in flexion. Her eyes showed conjugate deviation to the right, and there was intermittent coarse nystagmus. The pupils reacted to light and were equal but she showed no awareness of moving objects and she did not respond to auditory stimuli. Further examinations of the cerebrospinal fluid yielded normal results. She was finally discharged two and a half months after the onset of her illness-spastic and comatose, responding only to painful stimuli, and still prone to outbursts of prolonged crying.

\section{Virus Examination}

Apart from complement-fixation tests on sera from Cases 4 and 5 during life, pieces of frontal cortex, basal ganglia, cerebellum and medulla selected from Cases 2 and 3 were submitted to the Institute of Medical and Veterinary Science, Adelaide, for virus isolation. The material was inoculated into newborn and weaned mice, on to the rabbit's cornea, into embryonated eggs and HeLa cell tissue culture. No viruses were recovered.

\section{Discussion}

Scholz (1951) has published his findings in the brains of children dying after severe febrile convulsions, but his monograph is not available to the present writer. However, Meyer, Beck and Shepherd (1955) refer to his work in their paper describing the cerebral lesions in a boy of 9 years who suffered from status epilepticus a year before death. They found neuronal damage in various parts of the cerebral cortex, in the basal ganglia and cerebellar cortex. They accepted Scholz's view that brain lesions may be directly due to a convulsive disorder. Before considering the part played by the convulsions in the present cases it is first necessary to exclude the action of other well-recognized causes of cerebral disease.

In this series of cases, all the children were mentally and physically normal before the onset of the illness, except for Case 5 who has scurvy. The oldest child was 2 years. The precipitating illness was gastro-enteritis in two cases, upper respiratory infection in one, measles bronchopneumonia in one and undiagnosed in one. In all cases the temperature at the onset was above $102^{\circ} \mathrm{F}$. and in two cases reached $105^{\circ} \mathrm{F}$. The length of the convulsions was not easy to determine for two patients were already in the attack when found, but the shortest time was one hour (Case 3) and the longest time at least six hours. None of the patients recovered consciousness and as the residual twitchings faded various degrees of spastic paralysis appeared-complete quadriplegia in three cases, diplegia and hemiplegia each in one case.

The usual clinical diagnosis of encephalitis made in these cases was not confirmed by lumbar puncture, for the cerebrospinal fluid, apart from raised pressure in one instance, was normal. In Case 2 only bloody taps were obtained.

One of the children is still alive but pathological examination of the other four cases revealed damage to various parts of the cortex and basal ganglia. In Case 4 the frontal lobes and post-central gyri were involved bilaterally, all other areas examined being normal. The temporal and frontal lobes were severely damaged bilaterally in Cases 1 and 3, unilaterally in Case 2, but in this last case the superior temporal gyrus was selectively involved on the other side. Layer III was the most frequent and often only cortical layer showing disease, layers $\mathrm{V}$ and VI being involved next in frequency. The thalamus, caudate and amygdaloid nuclei were damaged in three cases, often severely, but in Case 2 unilaterally. The hippocampus contained lesions in three cases, severe in Cases 1 and 2. Lesions in the claustrum tended to be focal and small, unlike the diffuse 
lesions in the other nuclei. Damage of moderate severity was found in the lentiform nucleus in two cases. Only in Case 1 did the cerebellum show lesions, which consisted of small scattered groups of necrotic Purkinje cells. No lesions were found in the midbrain, pons or medulla. The choroid plexuses, cerebral arteries and dural sinuses were normal. There was no evidence of inflammatory reaction around the blood vessels or in the meninges, nor did the vessels contain thrombi or other significant abnormality, although in some cases they were somewhat dilated and in Case 2 greatly increased in number. No signs of perivascular demyelination or inflammation were found in the white matter and these were deliberately sought in the patient who had suffered from measles. The first four cases illustrate a progress of the changes from necrosis to disappearance of nerve cells, followed by astrocytic and microglial proliferation. An interesting finding in the three cases of shorter duration was the inability to impregnate astrocytes in areas of neuronal damage, associated with abnormal astrocytic forms in the border zones. In Case 4, however, of 18 weeks' duration, astrocytic proliferation was easy to demonstrate.

In Cases 2 and 3 tissue culture and animal inoculation failed to provide evidence for the presence of viruses in brain tissue removed at necropsy, and serum from Cases 4 and 5 gave negative complementfixation reactions. Case 5 is included, in spite of the lack of pathological proof, for the clinical findings are very similar to the other cases, and she is in a state of mental defectiveness and blindness most compatible with widespread cortical damage. An alternative diagnosis of haemorrhage due to scurvy is unacceptable for several reasons, the main ones being that there was no evidence of haemorrhage elsewhere and that the child had responded well to treatment. Moreover, haemorrhage great enough to damage the occipital and frontal cortex is most unlikely without signs of increased pressure or other abnormalities in the cerebrospinal fluid.

It seems clear, therefore, that these children were suffering from febrile convulsions, of a prolonged, severe type, and that brain damage occurred during the convulsion. The five cases appeared during a two-year period in a 300 -bed hospital so that the condition cannot be called rare. In contrast, Cary (1956), in a series of 100 cases of infantile febrile convulsions, found little evidence of serious cerebral impairment. This failure may well be due to exclusion of some grave cases on the grounds that they represent the convulsive prelude to encephalitis or other primarily cerebral disease. It is probable that some of the cases of mental deterioration after convulsions reported by Illingworth (1955) are examples of the same process described here. In my Case 4 a little less damage to the frontal lobes without involvement of the motor area could have led to mental changes alone.

The pathogenesis of the neuronal necrosis is not certain. Toxaemia from the infection is unlikely, for changes in the brain of the type and severity under discussion are not a feature in children dying of much more severe and prolonged infections. Direct action of microorganisms is a theory not supported by any of the findings. The two most acceptable theories are that either the nerve cells degenerate from the effects of the general anoxia which occurs during a convulsion or that there is a specific mechanism in the brain producing local anoxia. The unilateral character of the degeneration in Case 2 may be interpreted in favour of a local mechanism, although it is probable that individual cellular or lobar metabolic activity in the brain may influence the degree of susceptibility to general anoxia, e.g., the Purkinje cells illustrated in Fig. 3. This regional character of lesions resulting from what might reasonably be thought of as a generally acting disease process has been mentioned by Southcott and Fowler (1954) in relation to acute haemorrhagic leucoencephalitis. Against the participation of local vascular spasm in the causation of the changes is the conclusion of Penfield and Jasper (1954) that during an epileptic seizure the cortex, contrary to general opinion, does not blanche and that its blood flow is actually increased. In Case 1 the changes in the liver are very suggestive of anoxic degeneration, particularly in view of their central localization in the lobule, without inflammatory reaction. The lesions in the liver may. however, have been an effect of toxaemia, although I have never seen this phenomenon in other cases of equally severe and widespread bronchopneumonia due to Staphylococcus aureus. Anoxia as an important factor in centrilobular necrosis of the rabbit liver, particularly in conjunction with low blood pressure, has been shown by McMichael (1937) who concluded that due to the low oxygen tension in its mixed arterial and venous supply the liver is especially liable to suffer in states of general anoxia.

Several authors have reported the association of infantile infections with cerebral disease similar to the cases described here, but they have interpreted the association very differently. Woolf (1955) described the brain changes in three patients dying five, eight and 16 years after the sudden onset of a convulsive illness associated with infection. He thought the cerebral changes were due to dural 
sinus thrombosis, but, owing to the nature of his material, he did not have the opportunity to demonstrate the presence of old thrombi. Hallman, Hjelt and Tähka (1956) report the findings in two infants with gastro-enteritis. Both of their patients had convulsions early in the illness. These authors attribute the brain lesions to dehydration and the resulting vascular stasis. Kramer's clinical account (1954) of gastro-intestinal disturbance and cerebral damage includes four cases beginning acutely with convulsions.

A unique case is that of Crome (1952). Encephalopathy in the later stages of gastro-enteritis occurred in the absence of a history of convulsions. Crome attributed the cerebral changes to anoxia following dehydration and vascular stasis. The rarity of such an association is most unusual considering the extreme degrees of dehydration commonly produced by gastro-enteritis in infants. Nevertheless, the present cases demonstrate that similar lesions can occur in the absence of both gastro-enteritis and dehydration. The commonest factor found in all the reported examples, apart from Crome's case, is the presence of convulsions.

Putting aside the question of pathogenesis, several further aspects of the process are of importance. Meyer, Beck and Shepherd (1955) have interpreted the degeneration in the thalamus of their case as a retrograde effect, and therefore likely to throw light on the cortico-thalamic connexions. In Cases 1 and 2 the nerve cells of the thalamus and cortex were at the same stage of degeneration suggesting rather that the damage was simultaneous and not consecutive. Quite apart from this consideration these writers emphasize the importance of the lesions as foci for convulsive seizures in those patients who survive, particularly in reference to temporal lobe epilepsy. In their case and in three of the present cases the temporal lobes suffered severely. It is likely, moreover, that damage following febrile convulsions in early infancy may be the basis for many of the spastic conditions, such as Little's disease, infantile hemiplegia and other variants, with or without dementia, and of behaviour disorders.

\section{Summary}

Five children, aged up to 2 years, and previously normal except for one with scurvy, suffered from a prolonged convulsion at the beginning of febrile illnesses comprising gastro-enteritis (two cases), measles bronchopneumonia (one case), upper respiratory tract infection (one case) and unidentified (one case).

The patients remained unconscious after the convulsions, which lasted from one to six hours, but developed various spastic paralyses, and in four cases death occurred four days, 12 days, four weeks, and 18 weeks from the onset of the convulsion. Pathological examination revealed neuronal necrosis of variable distribution and severity in the cerebral cortex, basal ganglia and cerebellum, with no evidence of the direct action of microorganisms. The changes are considered to be the result of systemic anoxia of convulsive origin or, less likely, to be due to a specific mechanism acting locally in the brain. Alternative explanations put forward by other observers are discussed, and it is conceded that stagnation anoxia following dehydration in gastro-enteritis may be responsible on rare occasions. The importance of convulsive cerebral degeneration as a precursor of epilepsy and as the cause of infantile spastic and behaviour disorders is mentioned.

My thanks are due to the various members of the honorary medical staff who have kindly allowed me to use their case notes, and to the Board of Management of the Adelaide Children's Hospital for permission to publish these investigations. The virus examinations were kindly undertaken by Dr. Peter Warner and Dr. Alan Duxbury, of the Medical Research Division of the Institute of Medical and Veterinary Science, Adelaide.

\section{REFERENCES}

Cary, W. (1956). Med. J. Aust., 2, 254.

Crome, L. (1952). Archives of Disease in Childhood, 27, 468.

Hallman, N., Hjelt, L. and Tähka, H. (1956). Ann. paediat. Fenn., 2, 31 .

Illingworth, R. S. (1955). Archives of Disease in Childhood, 30, 529. Kramer, W. (1954). Ned. T. Geneesk., 98, 3631. Quoted by The Year Book of Neurology, Psychiatry and Neurosurgery, 1955-56 Series, p. 167.

McMichael, J. (1937). Quart. J. exp. Physiol., $27,73$.

Meyer, A., Beck, E. and Shepherd, M. (1955). J. Neurol. Neurosurg. Psychiat., 18, 24.

Penfield, W. and Jasper, H. (1954). Epilepsy and the Functional Anatomy of the Human Brain. Boston.

Scholz, W. (1951). Die Krampfschädigungen des Gehirns. Berlin. Quoted by Meyer, Beck and Shepherd, above.

Southcott, R. V. and Fowler, M. (1954). Med. J. Aust., 2, 65.

Woolf, A. L. (1955). J. ment. Sci., 101, 610. 